The University of San Francisco

USF Scholarship: a digital repository @ Gleeson Library |

Geschke Center

1993

\title{
Magnetic Multilayers: Quasiclassical Transport Via the Kubo Formula
}

Horacio E. Camblong

University of San Francisco, camblong@usfca.edu

Follow this and additional works at: http://repository.usfca.edu/phys

Part of the Physics Commons

\section{Recommended Citation}

Camblong, Horacio E., "Magnetic Multilayers: Quasiclassical Transport Via the Kubo Formula" (1993). Physics and Astronomy. Paper 15.

http://repository.usfca.edu/phys/15 


\section{AIP Appilied Physics}

Magnetic multilayers: Quasiclassical transport via the Kubo formula

Horacio E. Camblong and Peter M. Levy

Citation: J. Appl. Phys. 73, 5533 (1993); doi: 10.1063/1.353639

View online: http://dx.doi.org/10.1063/1.353639

View Table of Contents: http://jap.aip.org/resource/1/JAPIAU/v73/i10

Published by the American Institute of Physics.

\section{Related Articles}

Towards compact three-dimensional magnetoelectronics-Magnetoresistance in rolled-up $\mathrm{Co} / \mathrm{Cu}$ nanomembranes

Appl. Phys. Lett. 100, 022409 (2012)

Ultrasensitive anomalous Hall effect in $\mathrm{SiO} 2 / \mathrm{Fe}-\mathrm{Pt} / \mathrm{SiO} 2$ sandwich structure films

Appl. Phys. Lett. 100, 022404 (2012)

Hysteretic anomalous Hall effect in a ferromagnetic, Mn-rich Ge:Mn nanonet

Appl. Phys. Lett. 100, 012406 (2012)

Low field anisotropic colossal magnetoresistance in $\mathrm{Sm} 0.53 \mathrm{Sr} 0.47 \mathrm{MnO} 3$ thin films

J. Appl. Phys. 110, 123922 (2011)

Tuning magnetoresistance and exchange coupling in $\mathrm{ZnO}$ by doping transition metals

Appl. Phys. Lett. 99, 222503 (2011)

\section{Additional information on J. Appl. Phys.}

Journal Homepage: http://jap.aip.org/

Journal Information: http://jap.aip.org/about/about_the_journal

Top downloads: http://jap.aip.org/features/most_downloaded

Information for Authors: http://jap.aip.org/authors

\section{ADVERTISEMENT}

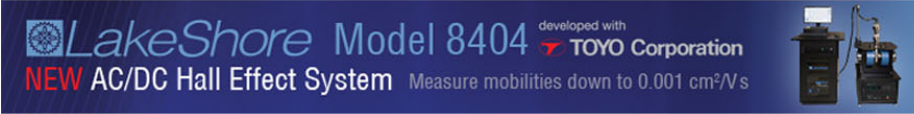




\section{Magnetic multilayers: Quasiclassical transport via the Kubo formula}

Horacio E. Camblong and Peter M. Levy

Department of Physics, New York University, New York, New York 10003

A real-space quantum approach, based on the Kubo formula, is used to describe the quasiclassical transport behavior of metallic multilayers. We emphasize the differences between the cases of current in the plane of the layers, for which size effects play a dominant role and current perpendicular to the planes, for which we provide several proofs that the so-called series resistor model holds for all length scales.

Giant magnetoresistance (GMR) in magnetic multilayers has created a great deal of excitement following its discovery in $1988,{ }^{1}$ due to its potential technological applications. It is generally agreed upon that this giant effect is not an ordinary galvanomagnetic effect (electron's dynamics), but it is due to the reorientation of antiferromagnetically coupled magnetic layers under the action of an external magnetic field. Spin-dependent scattering has been proposed as the basic underlying mechanism for the shortcircuit effect that yields the observed GMR, ${ }^{\prime}$ and several transport theories have been used to analyze the ensuing transport properties: (i) the quasiclassical (or Boltzmann) approach, ${ }^{2}$ (ii) the reciprocal space quantum approach, ${ }^{3}$ which extracts linear response coefficients from a model Hamiltonian with the Kubo formula in reciprocal space; (iii) the real-space quantum approach, which uses the Kubo formula in real space. ${ }^{40}$

Actually, the correct quantum-statistical calculation of linear transport properties, based on the Kubo formula, singles out quantum approaches (ii) and (iii) as more fundamental. However, the quasiclassical approach has been extensively used due to its simplicity, and because it seems to account for most of the observed features. We have recently obtained a real space solution ${ }^{5,6}$ of the quantum model first presented in Ref. 3, which is closely related to the solution based on the Boltzmann equation, and which relies upon a straightforward generalization of Kohn and Luttinger's impurity averages ${ }^{7}$ to multilayers. Moreover, we have shown that, for the characteristic parameters of metallic multilayers, an essentially quasiclassical behavior arises, and that the treatment of interfaces, in its simplest form, requires angle-dependent coherent transmission coefficients. ${ }^{6}$

In this paper we will further explore the connection between our real-space quantum approach and the Boltzmann approach, and we will apply our results to two geometries: current in the plane of the layers (CIP), and current perpendicular to the plane of the layers (CPP).

Our real space approach is based on a one-electron Hamiltonian with random impurity potentials. As the impurity average restores in-plane translational invariance, it is most convenient to work in the mixed $\left(\mathbf{k}_{\|}, z\right)$ representation. From the Dyson equation, the one-particle propagator $\mathscr{F}\left(z, z^{\prime}\right)$ satisfies

$$
\left[\frac{d^{2}}{d z^{2}}+k^{2}(z)\right] \mathscr{G}\left(z, z^{\prime}\right)=\delta\left(z-z^{\prime}\right)
$$

where $z$ is the growth direction,

$$
k(z)=\left[\vec{k}^{2}+i k_{F} / l(z)\right]^{1 / 2}
$$

is a complex wave number for propagation along the $z$ axis, $\bar{k}=\left[k_{F}^{2}-k_{\|}^{2}\right]^{1 / 2}$ and $\mathbf{k}_{\|}$are the effective $z$ and in-plane components of the conduction electron's momentum, and $l(z)$ is the local mean free path, which is related to the strength of the local scattering or imaginary part of the self-energy:

$$
\Delta(z)=-\operatorname{Im}\left[\sum(z)\right] \propto 1 / l(z) .
$$

In Refs. 5 and 6 we have solved Eq. (1) in each layer and matched the Green's functions of neighboring layers. In this paper we rederive the same result by solving Eq. (1) globally within a WKB approximation, that is, for small changes $|\Delta k|$ in $k(z):|\Delta k|<k_{f}$, which amounts to the quasiclassical limit $1 / l(z) \leqslant k_{F}$; then

$$
\begin{aligned}
\mathscr{G}\left(z, z^{\prime}\right) & \approx \frac{1}{2 i \bar{k}} \exp \left[i \int_{z<}^{z>} d z k(z)\right] \\
& \approx \frac{1}{2 i \bar{k}} \exp \left[i \vec{k}\left|z-z^{\prime}\right|-\frac{t}{2} \phi\left(z, z^{\prime}\right)\right],
\end{aligned}
$$

where $t=k_{F} / \vec{k}$, and

$$
\phi\left(z, z^{\prime}\right)=\int_{z<}^{z>} \frac{d z^{\prime \prime}}{l\left(z^{\prime \prime}\right)} \propto \int_{z<}^{z>} d z^{\prime \prime} \Delta\left(z^{\prime \prime}\right),
$$

with $z<(z>)$ being the smaller (larger) of $z$ and $z^{\prime}$. Equation (6) defines a symmetric two-point function that gives the decay in the one-particle propagator in terms of the average scattering between the two given points.

In particular, for an $N$-layered system, it is convenient to introduce the notation of Refs. 5 and 6: layers will be labeled by the subscript $j$ (with $j=1, \ldots, N$ ) and denoted by $\mathscr{L}_{j}=\left[z_{j-1}, z_{j}\right]$, and their thicknesses and local mean free paths will be $a_{j}=z_{j}-z_{j-1}$, and $l_{j}$, respectively. Also, defining $u_{j}=\left(z-z_{j-1}\right) / a_{j}$ (for $z$ in $\mathscr{L}_{j}$ ), one can introduce the following notation: $\phi\left(z, z^{\prime}\right)=\phi_{j, j^{\prime}}\left(u_{j}, u_{j^{\prime}}\right)$, for $z \in \mathscr{L}_{j}$ and $z^{\prime} \in \mathscr{L}_{j^{\prime}}$. Then, in the quasiclassical limit $k_{F} \rightarrow 1 / l_{j}$,

$$
\phi_{j, j^{\prime}}\left(u_{j}, u_{j^{\prime}}\right)=\Lambda_{j>} u_{j>}-\Lambda_{j<} u_{j<}+\alpha_{j<, j>-1}
$$

where $j<(j>)$ is the smaller (larger) of $j$ and $j^{\prime}, \Lambda_{j}=a_{j}$ ' $l_{j}$, and

$$
\alpha_{j<j>-1}=\sum_{j=j<}^{j>-1} \Lambda_{j} .
$$


As discussed in Refs. 5 and 6, the corresponding Green's function provides a good description of the transport behavior of metallic multilayers for which the total length $L$ is large compared with the mean free paths. In particular, it describes its local transport behavior everywhere except near external boundaries, and its global transport behavior, which consists in the dependence of the conductance with respect to the different length scales $a_{j}$ and $l_{j}$, namely, the size effects.

The ensuing linear transport theory is obtained by using the real-space Kubo formula. We will only consider experiments with a uniform external electric field, in which case the induced internal field remains uniform in the plane of the layers (due to in-plane translational invariance). Then, only two independent two-point conductivities are required: $\sigma^{(\mathcal{L})}\left(z, z^{\prime}\right)=\sigma_{z z}\left(\mathbf{k}_{\|}=0, z ; \mathbf{k}_{\|}^{\prime}=0, z^{\prime}\right)$ for current perpendicular to the plane of the layers (CPP), and $\left.\sigma^{\prime \|}\right)\left(z, z^{\prime}\right)=\sigma_{x x}\left(\mathbf{k}_{\|}=0, z ; \mathbf{k}_{\|}^{\prime}=0, z^{\prime}\right)$ for current in the plane of the layers (CIP). Then, the Kubo formula yields

$$
\sigma^{(i)}\left(z, z^{\prime}\right)=\frac{3 C_{D}}{4}\left\{E_{1}\left[\phi\left(z, z^{\prime}\right)\right]-E_{3}\left[\phi\left(z, z^{\prime}\right)\right]\right\}
$$

and

$$
\sigma^{(1)}\left(z, z^{\prime}\right)=\frac{3 C_{D}}{2} E_{3}\left[\phi\left(z, z^{\prime}\right)\right],
$$

where $\phi\left(z, z^{\prime}\right)$ is given by Eqs. (6) and (7), $C_{D}$ is the ratio between the local conductivity $\sigma_{\text {loc }}(z)$ and the local mean free path $l(z)$ of any layer [or, more precisely, $C_{D}=n e^{2} /$ $\left(m v_{F}\right)$, with $n$ being the density of conduction electrons], and $E_{n}(x)$ is the exponential integral function of order $n$.

These results are in complcte agreement with those obtained by solving the linearized Boltzmann equation in the relaxation time approximation,

$$
\mathbf{v} \cdot \nabla g(\mathbf{v}, z)+\frac{g(\mathbf{v}, z)}{\tau(z)}=(-e)\left(-\frac{\partial f^{(0)}}{\partial \epsilon_{\mathbf{k}}}\right) \mathbf{v} \cdot \mathbf{E}(z),
$$

where $f(\mathbf{v}, z)=f^{0}\left(\epsilon_{\mathrm{k}}\right)+g(\mathbf{v}, z)$ is the quasiclassical distribution function, and $f^{0}(\epsilon)$ is the equilibrium distribution function, which at low temperatures satisfies the equation $\partial f^{(0)} / \partial \epsilon_{\mathrm{k}}=-\delta\left(\epsilon_{F}-\epsilon_{\mathrm{k}}\right)$. It is convenient to rescale the distribution function according to $g(v, z) \propto \delta\left(\epsilon_{F}\right.$ $\left.-\epsilon_{\mathrm{k}}\right) h^{( \pm)}(z, t)$, where $t=v_{k} f\left|v_{z}\right|$ has the same meaning as before, and \pm refers to the sign of the $z$ component of the velocity the quasiclassical electrons. Then, calculation of the current density by integration of the velocity in reciprocal space allows a comparison with the current in the Kubo approach:

$\left.h^{( \pm)}(z, t) \propto \int_{I_{ \pm}} d z^{\prime}\left\langle\left[\mathscr{H}\left(z, z^{\prime}\right)\right]^{2}\right\rangle_{\mathrm{av}}\right|_{\bar{k}=k_{F^{\prime}}} E\left(z^{\prime}\right)$,

where $I_{+}\left(I_{-}\right)$is the interval to the left (right) of point $z$, $\mathscr{A}\left(z, z^{\prime}\right)$ is the density of states operator $A(\epsilon)=i\left[G_{\text {ret }}(\epsilon)\right.$ $\left.-G_{\text {adv }}(\epsilon)\right]$ in the mixed $\left(\mathbf{k}_{\|}, z\right)$ representation, ${ }^{6}$ and the integrand is subject to a "quasiclassical average" over a distance of the order of $k_{F}^{-1}$. Equation (11) admits a very simple physical interpretation: the present value of the distribution function for an electron reflects its past history, described in terms of quasiclassical trajectories (on the left for $k_{z}>0$, and on the right for $k_{z}<0$ ); this is precisely the content of the Chambers formula, ${ }^{8}$ which for multilayers rcads

$$
h^{( \pm)}[z(s), t] \propto \int d s^{\prime} \mathbf{v} \cdot \mathbf{E}\left[z\left(s^{\prime}\right)\right] \exp \left(-\int_{s<}^{s>} \frac{d s^{\prime \prime}}{l\left[z\left(s^{\prime \prime}\right)\right]}\right),
$$

where $s$ is the length along the quasiclassical electron's trajectory and $s<(s>)$ is the smaller (larger) of $s$ and $s^{\prime}$. Equation (12) yields two-point conductivities identical to Eqs. (8) and (9), because $d z^{\prime \prime}=d s^{\prime \prime} / t$ [see Eq. (6)].

It should be noticed that the main reason for the equivalence between the quantum and quasiclassical approaches, Eq. (11), is the validity of the approximation $k_{F}>[l(z)]^{-16}$

There are two limiting cases for which Eqs. (8), (9) have a simple form: ${ }^{9}$ (i) local limit, when $l_{j}<a_{j}$ for all layers; and (ii) self-averaging limit, when $l_{j} \gg a_{j}$ for all layers. In both cases the two-point conductivities resemble those of a homogeneous medium, but with different mean free paths: the local mean free path $l(z)$, and the selfaveraging mean free path $l$, defined via

$$
\frac{1}{l}=\frac{1}{L} \int \frac{d z}{l(z)}=\frac{1}{L} \sum_{j} \frac{a_{j}}{l_{j}},
$$

where $L$ is the total length of the sample. Equation (13) can be obtained directly from Eq. (6) and it yields the average reciprocal mean free path, (proportional to the average scattering in the medium).

Let us now consider the global or measurable conductivities, which can be calculated with

$\sigma_{\mathrm{CIP}}=\frac{(1 / L) \int d z j(z)}{E}=\frac{1}{L} \iint d z d z^{\prime} \sigma^{(\|)}\left(z, z^{\prime}\right)$,

for the CIP case (as the electric field is uniform), and

$$
\rho_{\mathrm{CPP}}=\frac{(1 / L) \int d z E(z)}{j}
$$

for the CPP case (as the current density is constant).

For the CIP case, let us consider a periodic multilayer, namely, a superlattice. For an $\boldsymbol{M}$-component superlattice extending along the whole $z$ axis, the global CIP conductivity is given by

$$
\begin{aligned}
\sigma_{\mathrm{CIP}}= & \sigma_{\mathrm{par}}-\frac{3 C_{D}}{4 T} \sum_{j_{0}^{\prime}>j_{\mathrm{o}}}^{\prime}\left(l_{j_{0}}-l_{j_{0}^{\prime}}\right)^{2} \int_{1}^{\infty} d t\left(\frac{1}{t^{3}}-\frac{1}{t^{3}}\right) \\
& \times\left(1-e^{-t \Lambda}\right)^{-1}\left(1-e^{-t \Lambda_{b_{0}}}\right)\left(1-e^{-t \Lambda} \Lambda_{\zeta_{0}^{\prime}}\right) \\
& \times\left(e^{\left.-t \alpha_{j<+1, j>-1}+e^{-t \alpha_{i j+1, j>+M-1}}\right),}\right.
\end{aligned}
$$

where $T=\Sigma^{\prime}, M$ $\Sigma^{\prime}$ indicates a restricted sum over fundamental period indices. We can interpret the limiting cases of Eq. (16) in terms of the resistor network analogy. In the local limit the different layers behave like classical or macroscopic resis- 
tors with local conductivity $\sigma_{\mathrm{loc}}(z)=C_{D} l(z)$ (or $\sigma_{\mathrm{loc}, j}$ $\left.=C_{D} l_{j}\right)$, and can be added in parallel for CIP: $\sigma_{\mathrm{ClP}}=\sigma_{\mathrm{par}}$ $=C_{D} l_{\text {par }}$, with

$$
l_{\text {par }}=\int d z l(z) / L=\sum_{j} a_{j} l_{j} / L .
$$

Instead, in the self-averaging limit they are effectively added in series, that is, $\sigma_{C \mathrm{Ip}}=\sigma_{\text {ser }}=C_{D} l_{\text {ser }}$, with

$$
l_{\mathrm{ser}}^{-1} \equiv \sum_{j}\left[a_{j} /\left(L l_{j}\right)\right]=l^{-1}
$$

(notice that, by definition, the self-averaging mean free path is identical to the series mean free path); this can be verified with the identity

$$
1-l_{\mathrm{par}} / l_{\mathrm{ser}}=-\sum_{j_{0}^{\prime}>j_{0}}\left[a_{j_{0}} a_{j_{0}^{\prime}}\left(l_{j_{0}}-l_{j_{0}^{\prime}}\right)^{2} /\left(T^{2} l_{j_{0}} l_{j_{0}^{\prime}}\right)\right]
$$

In general, Eq. (16) interpolates the CIP conductivity between two very different limiting cases, thus yielding very noticeable size effects.

On the other hand, for CPP, the equation of continuity implies that $\partial j / \partial z=0$, and the internal electric field is the solution $E(z)$ of the integral equation

$$
j=\int d z^{\prime} \sigma\left(z, z^{\prime}\right) E\left(z^{\prime}\right),
$$

for constant current density $j$. Now we set out to prove the following statement: The electric field distribution $E(z)$ which solves the integral Eq. (17) is inversely proportional to the local mean free path; that is, $j=\sigma_{\text {loc }}(z) E(z)$. In effect, the replacement $E(z) \propto 1 / l(z)$ converts $\mathrm{Eq}$. (17) into an identity, because [see Eqs. (6) and (9)]

$$
\int d z^{\prime} \frac{t}{l\left(z^{\prime}\right)} \exp \left[-t \int_{z<}^{z>} \frac{d z^{\prime \prime}}{l\left(z^{\prime \prime}\right)}\right]=2,
$$

which is valid for multilayers of length $L$ much larger than the local mean free path $l(z)$. Alternatively, one could just try the replacement $\partial g / \partial z=0$ in the Boltzmann equation, yielding an actual solution if $E(z) \propto 1 / l(z)$. With the result that $E(z) \propto 1 / l(z)$, it follows that the CPP resistivity, Eq. (15), is just given by the self-averaging mean free path, that is,

$$
\sigma_{\mathrm{CPp}}=C_{D} l=\sigma_{\text {ser }},
$$

which is solely determined by the average scattering in the medium. Therefore, the CPP resistivity is self-averaging and given by the series resistor model, not just for the local and self-averaging limiting cases, but for all length scales. This is the result that had been predicted by Zhang, ${ }^{10}$ and that has been found to be consistent with experimental results. ${ }^{11}$

In conclusion, we have clarified the connection existing between the Kubo and Boltzmann approaches for metallic multilayers, discussed size effects for CIP, and obtained the remarkable result that for CPP the series resistor model holds for all length scales.

\section{ACKNOWLEDGMENTS}

We would like to thank Dr. S. Zhang for his invaluable comments and critical remarks. This work was supported in part by the Office of Naval Research grant No. N0001491-J-1695.

'M. N. Baibich, J. M. Broto, A. Fert, F. Nguyen Van Dau, F. Petroff, P. Etienne, G. Creuzet, A. Friederich, and J. Chazelas, Phys. Rev. Lett. 61, 2472 (1988).

${ }^{2}$ R. E. Camley and J. Barnás, Phys. Rev. Lett. 63, 664 (1989).

${ }^{3}$ P. M. Levy, S. Zhang, and A. Fert, Phys. Rev. Lett. 65, 1643 (1990); S. Zhang, P. M. Levy, and A. Fert, Phys. Rev. B 45, 8689 (1992).

${ }^{4}$ A. Vedyayev, B. Dieny, and N. Ryzhanova, Europhys. Lett. 19, 329 (1992).

${ }^{5}$ H. E. Camblong and P. M. Levy, J. Magn. Magn. Mater. 121, 446 (1993).

${ }^{6}$ H. E. Camblong and P. M. Levy, Phys. Rev. Lett. 69, 2835 (1992).

${ }^{7}$ W. Kohn and J. M. Luttinger, Phys. Rev. 108, 590 (1957).

${ }^{8}$ R. G. Chambers, Proc. Phys. Soc. A 65, 458 (1952). See, for example, A. B. Pjppard, The Dynamics of Conduction Electrons (Gordon and Breach, New York, 1965), pp. 41-43.

${ }^{9}$ H. E. Camblong, S. Zhang, and P. M. Levy, Phys. Rev. B 47, 4735 (1993).

${ }^{10}$ S. Zhang and P. M. Levy, J. Appl. Phys. 69, 4786 (1991).

"W. P. Pratt, Jr., S.-F. Lee, J. M. Slaughter, R. Loloee, P. A. Schroeder, and J. Bass, Phys. Rev. Lett. 66, 3060 (1991); S. F. Lee, W. P. Pratt, Jr., R. Loloee, P. A. Schroeder, and J. Bass, Phys. Rev. B 46, 548 (1992). 